\title{
HMM and HSS Based Social Behavior of Intelligent Vehicles for Freeway Entrance Ramp
}

\author{
Guangming Xiong ${ }^{1}$, Yong $\mathrm{Li}^{1}$, Shiyuan Wang ${ }^{1}$, Xiaoyun $\mathrm{Li}^{1}$, Peng Liu ${ }^{2}$ \\ ${ }^{1}$ Intelligent Vehicle Research Center, Beijing Institute of Technology, 5 South \\ Zhongguancun Street, Haidian District, Beijing, China \\ ${ }^{2}$ Electrical and Computer Engineering Department, The Ohio-State University, \\ Columbus, OH, USA \\ xiongguangming@bit.edu.cn
}

\begin{abstract}
In this paper, a novel approach of intelligent vehicle to interact and cooperate with other human driving vehicles is proposed at the freeway entrance ramp scenario. The system consists of a Safety Alert Module and Vehicle Control Module. The Safety Alert System module includes intention estimation and conflict judgment. A two dimension Hidden Markov Model (2DHMM) is used to estimate social vehicle's driving intention in the intention estimation module. Then the conflict judgment module predicts the potential conflicts between the intelligent vehicle and social vehicle. Moreover, the hybrid state system (HSS) is introduced to control the intelligent vehicle while considering its decision making, states and dynamics. Co-simulation using PreScan and Simulink is conducted. The experiment results show that the intelligent vehicle performs well with a commendable social behavior in the freeway entrance ramp.
\end{abstract}

Keywords: social behavior; intelligent vehicle; hidden Markov model (HMM); hybrid state system (HSS); PreScan

\section{Introduction}

Intelligent vehicles have shown the potential to reduce traffic accidents and enhance the capacity and efficiency of the transportation system. Although intelligent vehicles have been rapidly developed in recently years, the traditional human-driven vehicles will continue to predominate in the short term. Therefore, intelligent vehicles have to cooperate and interact with social vehicles. Moreover, to enhance autonomous driving of intelligent vehicles in the real world, the decision-making system will benefit from the ability to socially cooperate with human-driven vehicles [1].

The related research often neglects social cooperation. For example, in the research on dynamic obstacle avoidance [2-3], the moving vehicles were only treated as dynamic obstacles. Their states were obtained by active detective sensors such as radar/LIDAR or camera. And the corresponding motion planner was based on the assumption that moving obstacles will keep constant velocity and drive along the road. However, if social vehicles are sheltered in the freeway entrance ramp and cannot be detected by the intelligent vehicle, there will be potential dangerous. On the other hands, the assumptions of dynamic vehicles do not accord with the reality that each vehicle's movement will affect surrounding vehicles' behavior. 
As a special category of robots, intelligent vehicle's social interaction is similar with human-robot interaction. Using the frameworks of POMDP, Broz et al., introduced human intention as a partially observable state in making decision [4]. So an intelligent vehicle can interact with another vehicle by estimating whether it wants to yield or not and perform proper actions. Wei et al., proposed an intention-integrated Prediction and Cost functionBased algorithm (iPCB) framework to realize "Socially cooperative driving" [1, 5]. However the vehicle dynamics were not taken into consideration in their simulation. In this paper, a novel approach to intelligent vehicle social behavior generation is proposed and implemented at the freeway entrance ramp scenario.

\section{System Structure}

The freeway entrance ramp is chosen in this paper as a traditional scenario in which the intelligent vehicle needs to conduct interaction behavior with surrounding human-driving vehicles, as shown in Figure 1. This paper assumes that the vehicles are running on roads and all the vehicles including the intelligent vehicle and other social vehicles are equipped with wireless communication [6] device independently. Therefore, they can broadcast their states such as position and velocity to other vehicles around them, which makes the intelligent vehicle be able to obtain the social vehicles' states in real time.

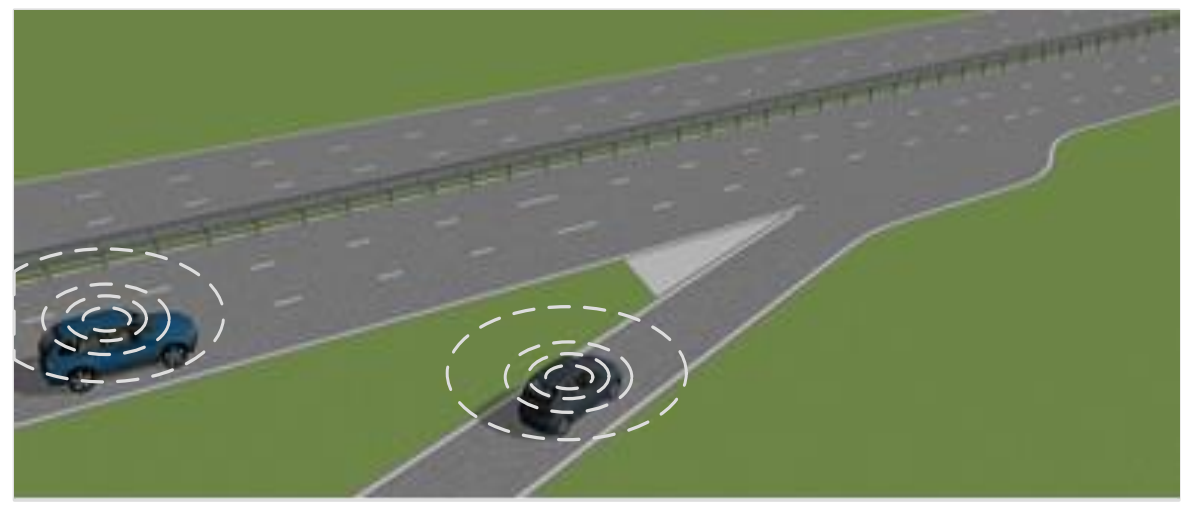

Figure 1. The Scenario of Freeway Entrance Ramp

The system structure of intelligent vehicle in this paper is developed, as shown in Figure 2. The system consists of Safety Alert Module and Vehicle Control Module. The Safety Alert Module module includes intention estimation and conflict judgment. The state of social vehicle is obtained through Vehicle-to-Vehicle (V2V) communication when the social vehicle is approaching the same lane as the intelligent vehicle. Firstly, it integrates the states of intelligent vehicle and social vehicles to predict whether there would be a conflict between them. If the collision is forecasted, the driver's intention of social vehicle will be estimated and used to control the vehicle. The vehicle control module consists of high level control, information process and low level control. High level control module generates strategies based on the intention of social vehicles. Information process module transforms the command from high level control module to control of low level control module and collects the information from low level control module. Considering the discrete decision-making and the continuous states of vehicle, the hybrid state system (HSS) is used in this paper to express the intelligent vehicle system dynamic. 


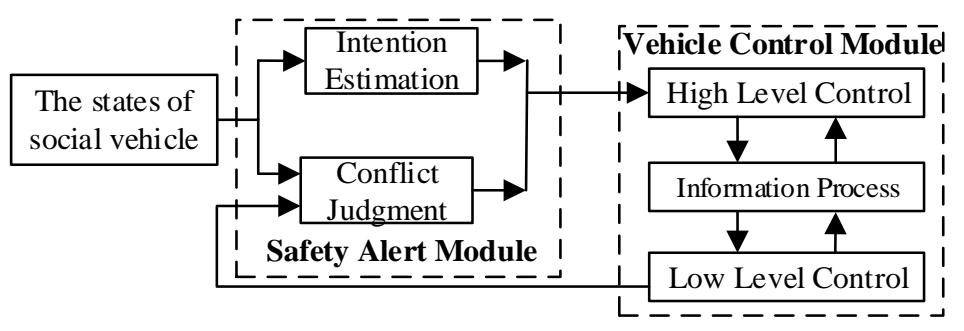

Figure 2. System Structure of the Intelligent Vehicle

\section{Driver Intention Estimation}

The Hidden Markov Model (HMM) [7] is used to estimate the social vehicle's intention in this paper. Firstly, the velocity and acceleration information of lots of vehicles is collected when these vehicles run cross the freeway entrance ramp. Then the information is clustered into many discrete training data. After that, the Baum-Welch method [8] is used to estimate the maximum likelihood model parameters for different driver intentions. Then using these parameters, the driver's intention can be estimated. The whole process is illustrated in Figure 3. After the data processing, the intelligent vehicle can get a discrete observation sequence. The probabilities of different driver intention are calculated using the forward algorithm [8]. The highest likelihood probability corresponds to the estimated driver intention.

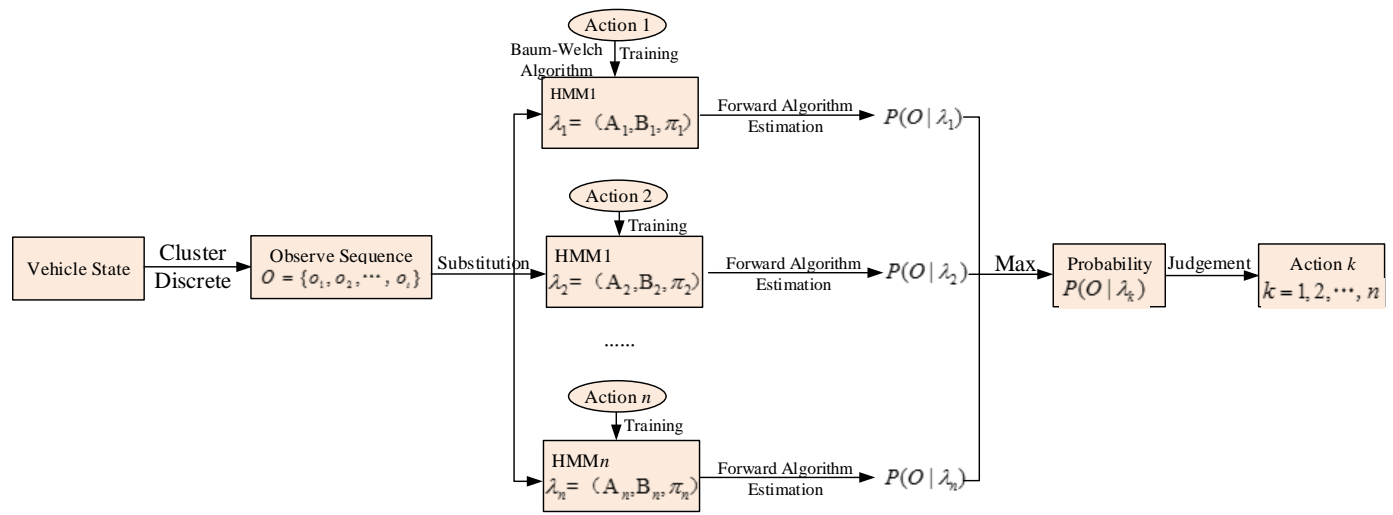

Figure 3. The Process of Driver Intention Estimation

\subsection{Hidden Markov Models}

A discrete HMM consists of a set of N finite "hidden" states, $S=\left\{s_{1}, s_{2}, \ldots, s_{N}\right\}$, and a set of M observable symbols per state, $\boldsymbol{O}=\left\{o_{1}, o_{2}, \cdots, o_{T}\right\}$. In this paper, the hidden states are the vehicle's driving state which cannot be obtained directly; and the observation symbols are the discrete observation information. $R_{t}$ and $U$, denote the hidden state and observation symbol at time t respectively [8]. Other elements of an HMM are defined as follows:

1)The state transition probabilities, $\boldsymbol{A}=\left\{a_{i j}\right\}$, where

$$
a_{i j}=P\left\{R_{t+1}=s_{j} \mid R_{t}=s_{i}\right\}, 1 \leq i, j \leq N
$$

2)The observation symbol probability distribution, $\boldsymbol{B}=\left\{b_{i j}\right\}$, where 


$$
b_{i j}=P\left\{U_{t}=v_{j} \mid R_{t}=s_{i}\right\}, 1 \leq k \leq M
$$

3)The initial state distribution, $\pi=\left\{\pi_{i}\right\}$, where

$$
\pi_{i}=P\left\{R_{1}=s_{i}\right\}, 1 \leq i \leq N
$$

Therefore, a HMM can be represented with

$$
\lambda=(N, M, \boldsymbol{A}, \boldsymbol{B}, \boldsymbol{\pi})
$$

\subsection{Forward Algorithm}

Calculating the probability of the observation sequence $\boldsymbol{O}=\left\{o_{1}, o_{2}, \cdots, o_{T}\right\}$ can be seen as an evaluation when given the model $\lambda=(\boldsymbol{A}, \boldsymbol{B}, \boldsymbol{\pi})$. The most straightforward way of doing this is through enumerating every possible state sequence of length $T$. But this calculation method is of huge computation; its time complexity is $2 T N^{T}$. To reduce the time complexity the forward algorithm is used in this paper.

Considering the forward variable $\alpha_{t}(i), \alpha_{t}(i)=P\left(o_{1} o_{2} \cdots o_{t}, R_{t}=s_{i} \mid \lambda\right), 1 \leq i \leq T$, the procedure of forward algorithm is shown as follows:

1) Initialization:

$$
\alpha_{1}(i)=\pi_{i} b_{i}\left(o_{1}\right), 1 \leq i \leq T
$$

2) Recursion:

$$
\alpha_{t+1}(j)=\left[\sum_{i=1}^{N} \alpha_{t}(i) a_{i j}\right] b_{j}\left(o_{t+1}\right), 1 \leq i \leq T, 1 \leq j \leq N
$$

3) Result:

$$
P(\boldsymbol{O} \mid \boldsymbol{\lambda})=\sum_{i=1}^{N} \alpha_{T}(i)
$$

(1)

The time complexity of forward algorithm is $\mathrm{N}^{2} \mathrm{~T}$.

\subsection{Two-dimension HMM}

The driver's driving behavior has its similar rule in specific driving condition. The driving intention can be reflected by lots of vehicle information [9-10]. In this paper the vehicle information can be represented by

$$
O(t)=\{x(t), y(t)\}
$$

where $x(t)$ represents the social vehicle's velocity, and $y(t)$ represents its acceleration value [11-12].

With the vehicle information data, the parameters of the HMMs which indicate two kinds of driver intention_-yield or not yield are trained. The information data used to train are two-dimensional, so a two-dimensional HMM is introduced to express the state transition.

A two-dimensional HMM (2D HMM) can be represented by a tuple $\lambda=\left(\mathrm{N}, \mathrm{M}, \pi, \mathbf{A}, \mathbf{B}_{1}\right.$, $\mathbf{B}_{2}$ ) , where N, M, $\pi$ and $\mathbf{A}$ are the same as the standard HMM. $\mathbf{B}_{1}, \mathbf{B}_{2}$ are the observation symbol probability distribution of the vehicle's speed and acceleration. The output probability of the two-dimensional HMM can be calculated as the product of the output probability of the signal for each dimension. The iterative formula of forward variable in standard HMM is modified as: 


$$
\alpha_{t+1}(j)=\left\lceil\sum_{i=1}^{N} \alpha_{t}(i) a_{i j}\right\rceil \prod_{l=1}^{2} b_{j}\left(\mathrm{O}_{t+1}(l)\right)
$$

For the evaluation of the model parameters, the revaluation formula of $\mathbf{B}$ is modified as:

$$
\bar{b}_{j}^{(l)}(k)=\operatorname{count}\left(k^{(l)} \mid j\right) / \operatorname{count}(j)
$$

Where count $\left(k^{(l)} \mid j\right)$ represents the expectation of state $v_{k}$ in the observation symbol set $l$ when the hidden state is $s_{j}, l=1,2$.

\section{Decision-making Model}

Using HMM to estimate intention of social vehicles, it's sometimes hard to get an explicit value, and it may lead to similar possibilities of yielding and not yielding, so as to cause terrible traffic accidents on the freeway. To solve the problems above, intelligent vehicles' interactive operation of social behavior, like speeding up or honking, is put forward. So it's easy for social vehicles to tell whether intelligent vehicles will yield or not through the explicit information. Repeating the previous process of intention judgment, intelligent vehicles can realize the interaction of social behavior with social vehicles and pass the freeway entrance ramp quickly without accidents. Figure 4 shows the flowchart of decisionmaking controller.

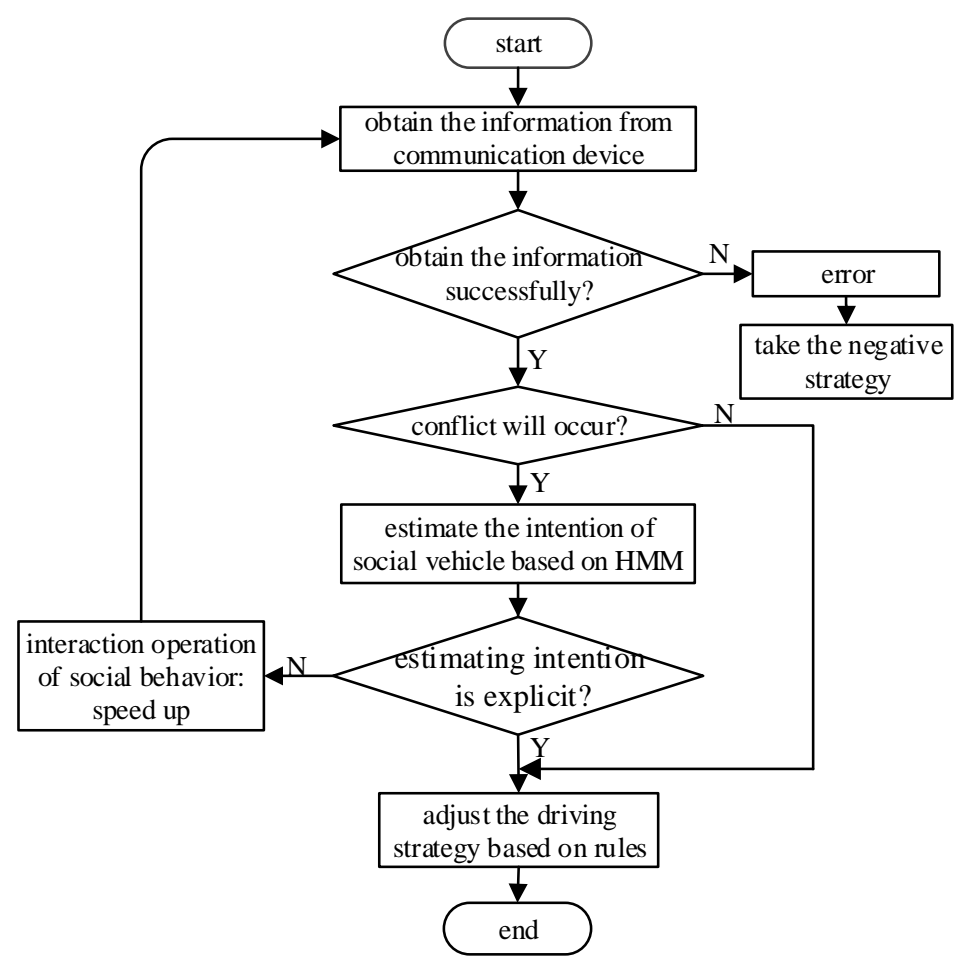

Figure 4 The Flowchart of Decision-making Controller

After identifying social vehicles' intention, intelligent vehicles can tell whether a collision will occur while keeping the current speed. The driving states will be adjusted by rule-based FSM if the collision may occur, as shown in Figure 5. 


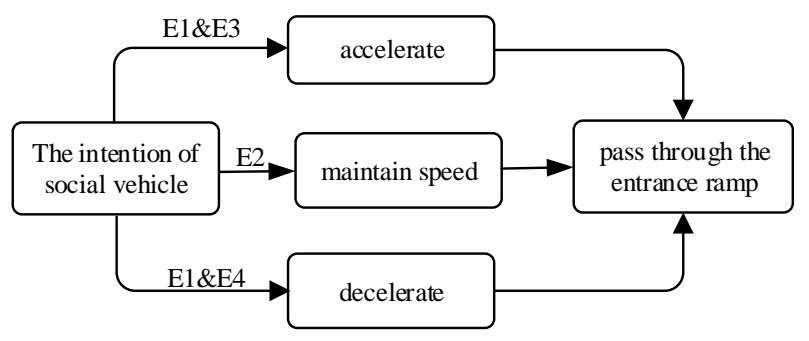

Figure 5. FSM Based on Rules

In Figure 5,

E1: represents the collision will occur if intelligent vehicles keep the current speed.

E2: represents intelligent vehicles can pass the freeway entrance ramp safely with the current speed.

E3: represents social vehicles will yield.

E4: represents social vehicles will not yield.

\section{Hybrid State System}

Intelligent vehicles' control system guarantees the vehicle driving safely based on their ego states information and social vehicles' driving intention derived from the Safety Alert Module. Intelligent vehicles' driving states and social vehicles' driving intention are all discrete states, but the states such as speed and heading, which are used to control the intelligent vehicle, all belong to continuous states. What's more, the changes of continuous states also in turn affect the discrete states. Therefore, the system architecture of intelligent vehicles is a kind of Hybrid state system [13-14], which combines continuous state system and discrete state system together, as shown in Figure 6.

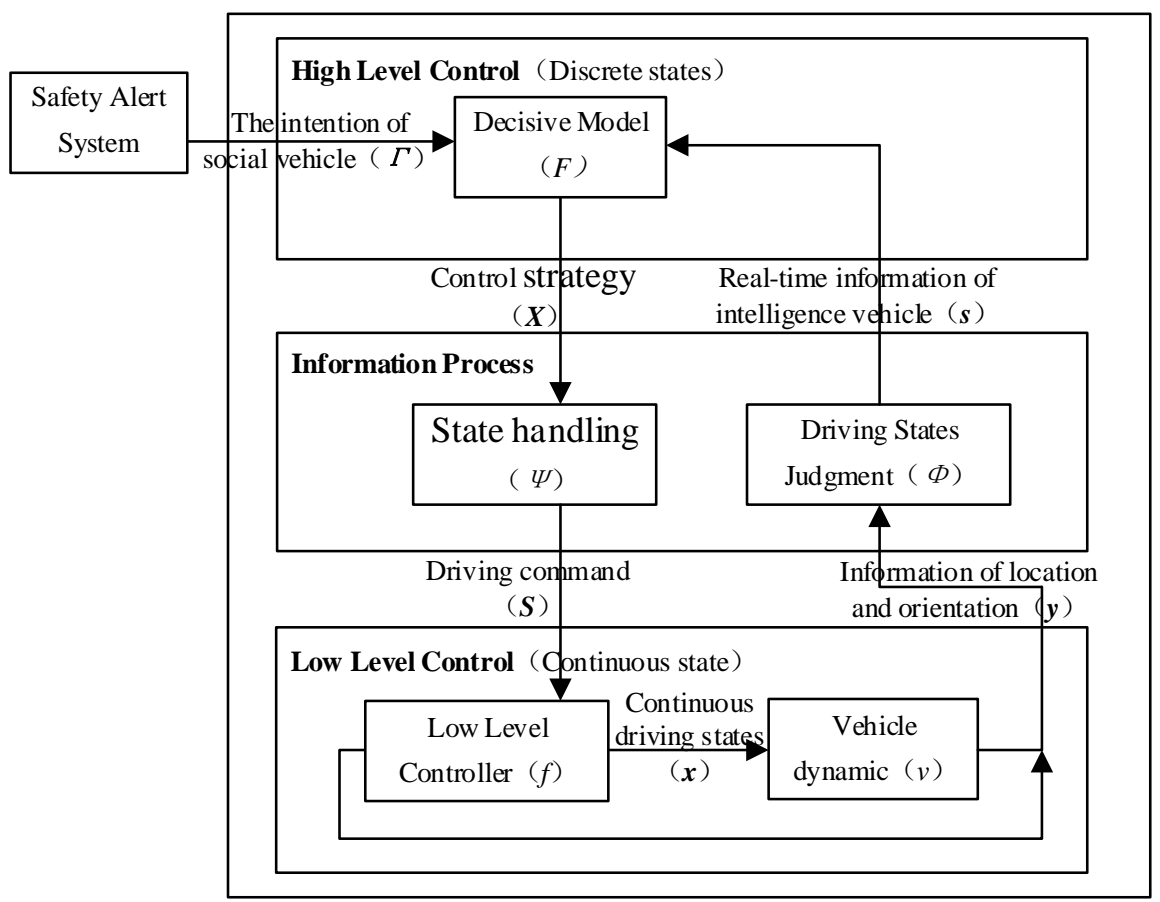

Figure 6. Hybrid State System 
As shown in Figure 6, the working process of the whole system architecture is as follows:

a) The high level control model carries out the control strategy based on social vehicles' intention $\Gamma$, intelligent vehicles' information $s$, and the control strategy $X$ by rule-based FSM;

b) State handling $\Psi$ in the information process converts the control strategy into driving command $s$ (route and speed), and pass it on to the low level controller;

c) The low level controller obtains the new continuous driving states based on driving command $S$ and the position \& orientation information $y$ so as to realize the control of intelligent vehicles.

\section{Simulation Experiments}

The co-simulation with PreScan 6.7.0 \& Matlab-Simulink is made to verify the proposed approach. PreScan 6.7.0 is used to build the simulation environment and Matlab-Simulink is used to program the control algorithm. The system structure based on Simulink is showed in Figure 7.

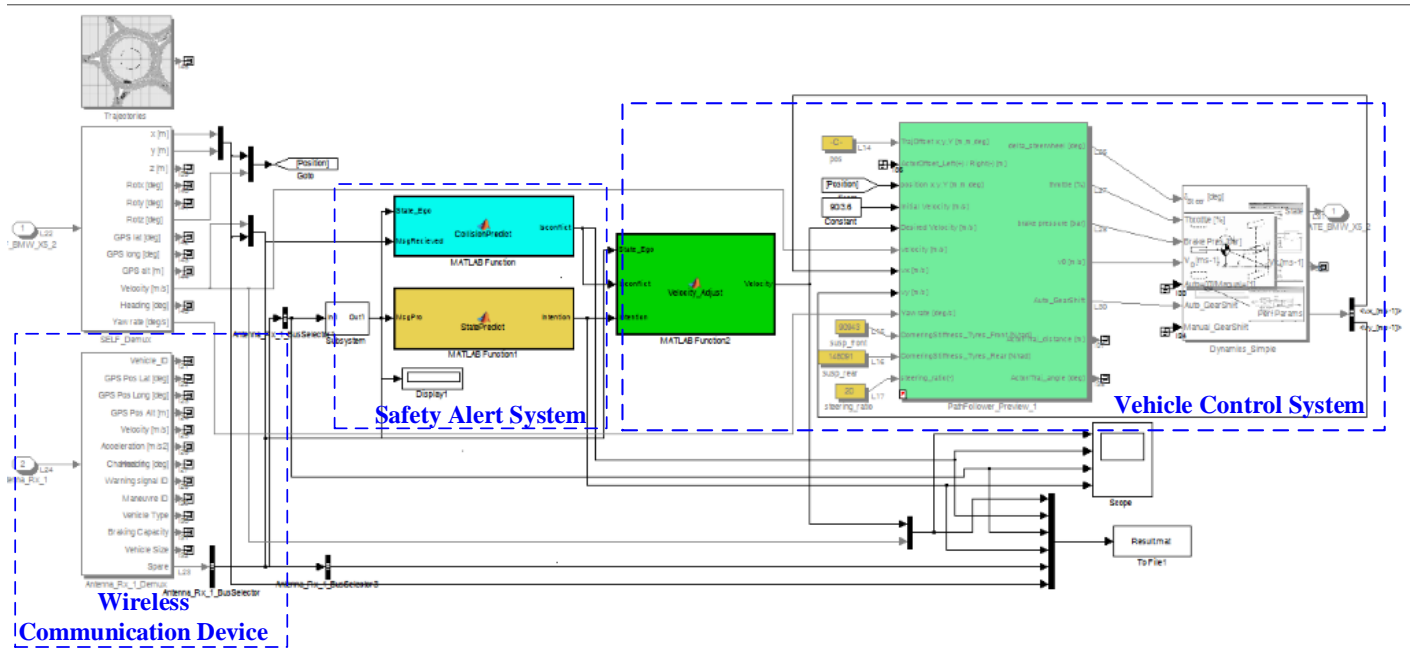

Figure 7. System Structure Based on Simulink

\subsection{Driving Intention Estimation of Social Vehicle}

The velocity and acceleration of the social vehicle is used to estimate driver's intention in the simulation. Cooperation with the speeding intelligent vehicle on the freeway, social vehicle's driving intention $i$ is defined as two values-yield and not yield. The parameters of different driving intentions corresponding the 2DHMM, $\left[\boldsymbol{\pi}, \boldsymbol{A}, \boldsymbol{B}_{1}, \boldsymbol{B}_{2}\right]$, are trained from vast experiment data. During the simulation, the data, which is generated from several random functions that represents different driving intentions, is classified into different clusters. For example, the velocity is classified into 6 clusters, and the acceleration into 3 clusters in this experiment.

With the training data, the parameters of different driving intention models $\lambda_{i}$ are trained based on the Baum-Welch algorithm. Consequently, the corresponding 2DHMM $\lambda_{i}=\left(\pi_{i}, \boldsymbol{T r a n s}_{i}, \boldsymbol{E} \boldsymbol{m} i \boldsymbol{s}_{i 1}, \boldsymbol{E} \boldsymbol{m} i s_{i 2}\right)$, is obtained, where $i$ represents different driving intention of social vehicle. When $i=$ unyeild, the parameters of the corresponding 2DHMMs are as follows: 


$$
\begin{aligned}
& \text { Trans } \text { unyield }=\left[\begin{array}{ll}
0.58056 & 0.41944 \\
0.89145 & 0.10855
\end{array}\right] \\
& \boldsymbol{E} \boldsymbol{m} \boldsymbol{i s}_{\text {unyield } 1}=\left[\begin{array}{llllll}
0.11181 & 0.17000 & 0.19786 & 0.20594 & 0.20080 & 0.11359 \\
0.89145 & 0.17149 & 0.19777 & 0.20464 & 0.19843 & 0.11184
\end{array}\right] \\
& \boldsymbol{E} \boldsymbol{m} \boldsymbol{i s}_{\text {unyield } 2}=\left[\begin{array}{lll}
0.00050 & 0.21601 & 0.78399 \\
0.00120 & 0.21968 & 0.78032
\end{array}\right]
\end{aligned}
$$

When $i=$ yeild, the parameters are as follows:

$$
\begin{aligned}
& \text { Trans }_{\text {yield }}=\left[\begin{array}{ll}
0.08173 & 0.91827 \\
0.41391 & 0.58609
\end{array}\right] \\
& \boldsymbol{E} \boldsymbol{m} \text { is }_{\text {yield } 1}=\left[\begin{array}{llllll}
0.11561 & 0.20361 & 0.20755 & 0.19780 & 0.16835 & 0.10709 \\
0.11177 & 0.19840 & 0.20468 & 0.19762 & 0.17164 & 0.11588
\end{array}\right] \\
& \boldsymbol{E} \boldsymbol{m} \boldsymbol{i s}_{\text {yield } 2}=\left[\begin{array}{lll}
0.78353 & 0.21647 & 0.00035 \\
0.78219 & 0.21781 & 0.00124
\end{array}\right]
\end{aligned}
$$

When the intelligent vehicle approaches the freeway entrance ramp zone which is $200 \mathrm{~m}$ apart from entrance ramp, the ID number of the vehicle is obtained. At the same time, the communication device on the intelligent vehicle is working for receiving social vehicles' information and broadcasting ego states. Once the same entrance ramp ID number of social vehicle is matched, its vehicle driving states such as location \& velocity are obtained. Then the safety alert module of intelligent vehicle starts working. To estimate the social vehicle's intention, its velocity and acceleration are sampled at $10 \mathrm{~Hz}$. Then a group of velocity and acceleration, shown in the Figure 8, would be classified into a group of observation sequence

$\boldsymbol{O}=\left[\begin{array}{cccc}o_{v_{1}} & o_{v_{2}} & \cdots & o_{v_{20}} \\ o_{a_{1}} & o_{a_{2}} & \cdots & o_{a_{20}}\end{array}\right]$

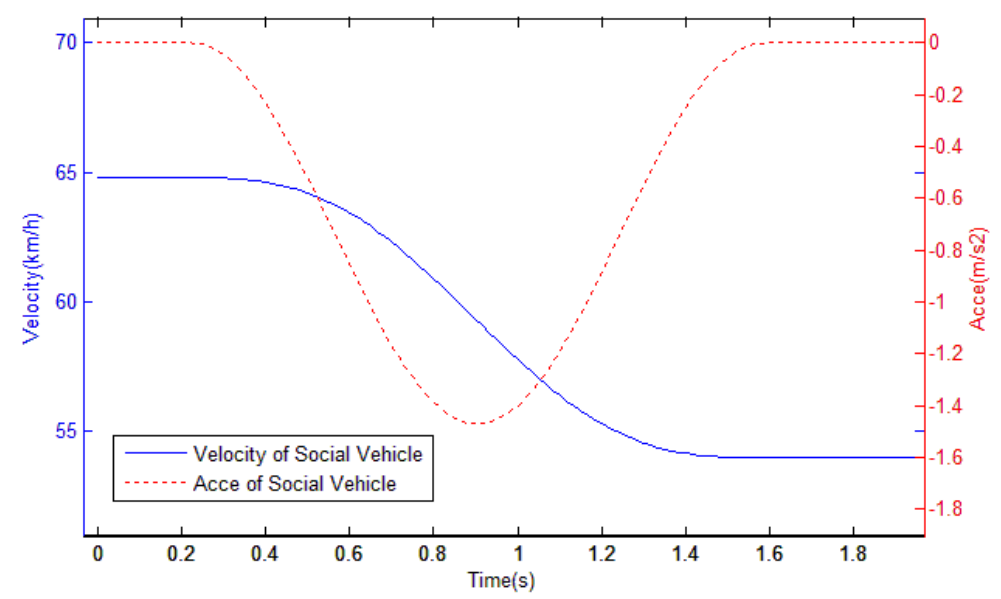

Figure 8. State Information of Social Vehicle

Forward algorithm is used to compute the probabilities, where $p_{\text {yielding }}=P\left(\boldsymbol{O} \mid \lambda_{\text {yielding }}\right) \quad$ represents the social vehicle's yielding probability and 
$p_{\text {unyeilding }}=P\left(\boldsymbol{O} \mid \lambda_{\text {unyeilding }}\right)$ represents the social vehicle's unyielding probability. Due to very small probability value, it is processed through a logarithmic function.

\section{Table 1. The Probabilities of Observation Sequence $o$ Under Different Intentions}

\begin{tabular}{|c|c|c|}
\hline Intention & Value & Estimate result \\
\hline yielding & -63.8394 & \multirow{2}{*}{ yield } \\
\hline unyielding & -141.1482 & \\
\hline
\end{tabular}

Comparing the two values, the social vehicle's driving intention is estimated as "yield".

\subsection{Intelligent Vehicle's Interaction with Social Vehicles}

When the conflict may occur and the social vehicle's driving intention is also estimated, the intelligent vehicle would interact with the social vehicle. In the simulation experiments, the initial velocity of intelligent vehicle was set as $90 \mathrm{~km} / \mathrm{h}$, and the states of social vehicle are set in the Figure 9(a). Then the corresponding intention estimate, conflict judgment and adjusted velocity of the intelligent vehicle are presented as in the Figure 9(b) (d) in real time respectively.
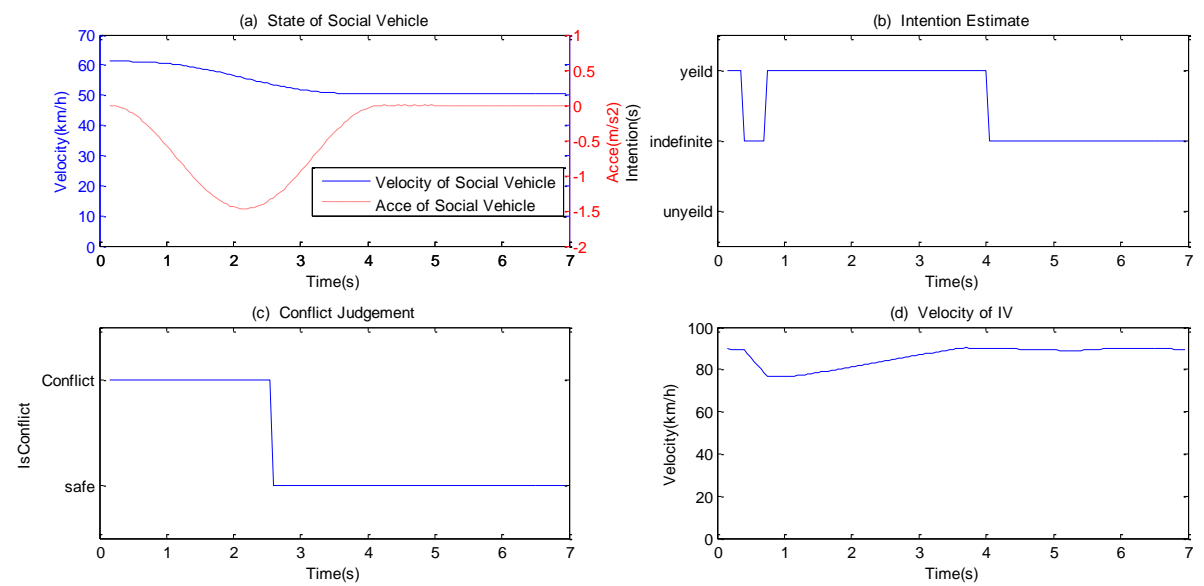

\section{Figure 9. Simulation Scenario 1---The Social Vehicle "yielding"}

From the figure we can analyze that, after receiving the state information of social vehicle, the intelligent vehicle has shown a satisfactory interaction performance. While $t=0 \mathrm{~s}$, as the intention estimation of social vehicle is indefinite and there would be a potential conflict, the intelligent vehicle takes the negative strategy - speed down. While $\mathrm{t}=0.4 \mathrm{~s}$, there also would be a potential conflict while the intention of social vehicle is estimated as "yield"----give way for the vehicle on the freeway, the intelligent vehicle begins to speed up until the conflict was eliminated at $\mathrm{t}=2.2 \mathrm{~s}$.

Similarly, when the states of social vehicle are set in the Figure 10(a), the corresponding interaction is shown in Figure 10(b) (d). There also would be a potential conflict at $t=0.8 \mathrm{~s}$ while the intention of social vehicle is estimated as "unyielding"----maybe no vehicle is detected for the social vehicle, the intelligent vehicle continues to speed down until the 
conflict is eliminating at $\mathrm{t}=2.8 \mathrm{~s}$. Then the intelligent vehicle accelerates to reach its original velocity.
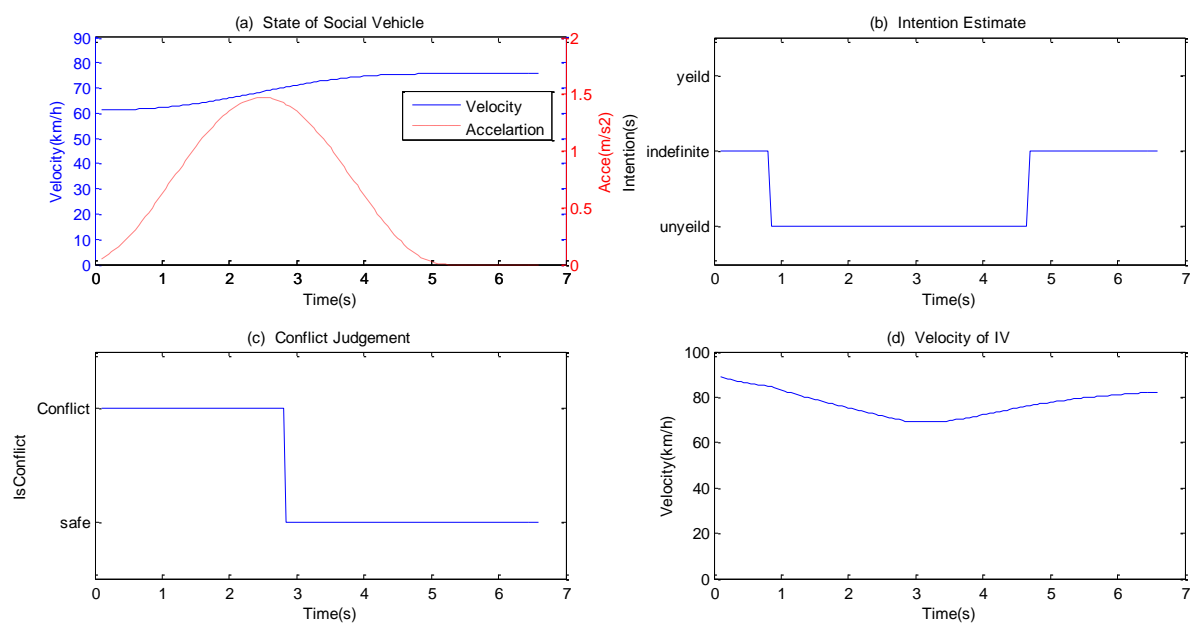

Figure 10. Simulation Scenario 2---The Social Vehicle "unyielding"

When the intention of social vehicle is indefinite for a long time and the safety is considered in the freeway, it would not be the advisable way for the intelligent vehicle to speed down all the times. Then another interaction signal such as the acceleration is given to the social vehicle, and the intelligent vehicle would estimate the social vehicle's further intention. If it is "yield", the intelligent vehicle will speed up to pass through the freeway ramp; else, the intelligent vehicle will speed down until there is no conflict.

\section{Conclusions}

For the freeway entrance ramp scenario, a novel approach to intelligent vehicles social behavior generator was proposed in this paper. Using this approach, an intelligent vehicle is capable of estimating the social vehicle's driving intention based on wireless data by an HMM. Moreover, HSS was used to control the intelligent vehicle considering discrete driving strategies, consecutive states and vehicle dynamics. In this way, the intelligent vehicle has better cooperative and interactive performance with social vehicles. The 2DHMM was established to realize intelligent vehicle's social behavior in the freeway entrance ramp. In the simulation, the statistical data which represent different intentions was used to train the parameters of 2DHMM. Using these models, the intelligent vehicle understands the social vehicle's driving intentions and performs well with a commendable social behavior in the scenario.

\section{Acknowledgements}

This work was supported by the National Natural Science Foundation of China (Grant No. 91120010). 


\section{References}

[1] J. Wei, J. M. Dolan and B. Litkouhi, "Intelligent vehicle social behavior for freeway entrance ramp management', Intelligent Vehicles Symposium (IV), 2013 IEEE, (2013) June 23-26, Gold Coast, Australia.

[2] M. Buehler, K. Iagnemma and S. Singh, "The DARPA urban challenge: intelligent vehicles in city traffic", springer, (2009).

[3] S. Kammel, J. Ziegler, B. Pitzer, M. Werling, T. Gindele, D. Jagzent, J. Schröder, M. Thuy, M. Goebl and F. V. Hundelshausen, "Team AnnieWAY's intelligent system for the 2007 DARPA Urban Challenge", Journal of Field Robotics 25, vol. 9, (2008).

[4] F. Broz, "Planning for human-robot interaction: representing time and human intention", ProQuest, (2008).

[5] J. Wei, J. M. Dolan and B. Litkouhi, "A prediction-and cost function-based algorithm for robust intelligent freeway driving”, Intelligent Vehicles Symposium (IV), 2010 IEEE, (2010) June 21-24, San Diego CA, USA

[6] J. Mikulski, "Using telematics in transport, Transport Systems Telematics", Springer, (2011).

[7] L. He, C. Zong and C. Wang, "Driving intention recognition and behaviour prediction based on a double-layer hidden Markov model", Journal of Zhejiang University SCIENCE C 13, vol. 3, (2012).

[8] L. R. Welch, "Hidden Markov models and the Baum-Welch algorithm", IEEE Information Theory Society Newsletter 53, vol. 4, (2003).

[9] X. Li, M. Parizeau and R. Plamondon, "Training hidden markov models with multiple observations-a combinatorial method", Pattern Analysis and Machine Intelligence, IEEE Transactions on 22, vol. 4, (2000).

[10] K. Mizutani and T. Omori, “A feasibility study of driver's cognitive process estimation from driving behavior", IEEJ Transactions on Electronics, Information and Systems 125, (2005).

[11] X. Meng, K. K. Lee and Y. Xu, "Human driving behavior recognition based on hidden markov models", International Conference on Robotics and Biomimetics, 2006 IEEE, (2006) December 17 -20 , Kunming, China.

[12] C. Liu, "Research on driving intention identification based on hidden markov model", Master's Thesis, Jilin University, (2011), (in chinese).

[13] A. Kurt, and U. Ozguner, Hybrid state system development for intelligent vehicle control in urban scenarios. Proceedings of the IFAC 2008 World congress, (2008) July 6-11, Seoul, Korea.

[14] A. Kurt, J.L. Yester, Y. Mochizuki, and U. Ozguner, IEEE. Hybrid-state driver/vehicle modelling, estimation and prediction. Intelligent Transportation Systems (ITSC), 2010 13th International IEEE Conference on, (2010) Sept 19-22, Madeira Island, Portugal. 
International Journal of Control and Automation Vol.7, No.10 (2014) 